\title{
Simple, Rapid and Sensitive Portable Molecular Diagnosis of SFTS Virus Using Reverse Transcriptional Loop-Mediated Isothermal Amplification (RT-LAMP)
}

\author{
Yun Hee Baek ${ }^{1 \dagger}$, Hyo-Soon Cheon ${ }^{1 \dagger}$, Su-Jin Park ${ }^{1 \dagger}$, Khristine Kaith S. Lloren ${ }^{1}$, Su Jeong Ahn ${ }^{1}$, \\ Ju Hwan Jeong ${ }^{1}$, Won-Suk Choi ${ }^{1}$, Min-Ah Yu ${ }^{1}$, Hyeok-il Kwon ${ }^{1}$, Jin-Jung Kwon ${ }^{1}$, Eun-Ha Kim ${ }^{1}$, Young-il Kim ${ }^{1}$, \\ Khristine Joy C. Antigua ${ }^{1}$, Seok-Yong Kim ${ }^{1}$, Hye Won Jeong ${ }^{2,3}$, Young Ki Choi ${ }^{1 *}$, and Min-Suk Song ${ }^{1 *}$ \\ ${ }^{1}$ Department of Microbiology, ${ }^{2}$ Department of Internal Medicine, Chungbuk National University College of Medicine and Medical Research \\ Institute, Cheongju 28644, Republic of Korea \\ ${ }^{3}$ Department of Internal Medicine, Chungbuk National University Hospital, Cheongju 28644, Republic of Korea
}

\author{
Received: June 12, 201 \\ Revised: September 6, 2018 \\ Accepted: September 12, 2018 \\ First published online \\ September 20, 2018 \\ ${ }^{*}$ Corresponding authors \\ Y.-K.C. \\ Phone: +82-43-261-3384; \\ Fax: +82-43-272-1603; \\ E-mail: choiki55@chungbuk.ac.kr \\ M.-S.S. \\ Phone: +82-43-261-3778; \\ Fax: +82-43-261-3778; \\ E-mail: songminsuk@chungbuk.ac.kr \\ ${ }^{+}$These authors contributed \\ equally to this work. \\ pISSN 1017-7825, eISSN 1738-8872 \\ Copyright (C) 2018 by \\ The Korean Society for Microbiology \\ and Biotechnology
}

Recently, human infections caused by severe fever with thrombocytopenia syndrome virus (SFTSV), which can lead to fatality, have dramatically increased in East Asia. With the unavailability of vaccines or antiviral drugs to prevent and/or treat SFTSV infection, early rapid diagnosis is critical for prevention and control of the disease. Here, we report the development of a simple, rapid and sensitive portable detection method for SFTSV infection applying reverse transcription-loop mediated isothermal amplification (RT-LAMP) combined with one-pot colorimetric visualization and electro-free reaction platform. This method utilizes a pocket warmer to facilitate diagnosis in a resource-limited setting. Specific primers were designed to target the highly-conserved region of L gene of SFTSV. The detection limit of the RT-LAMP assay was approximately $10^{\circ}$ viral genome copies from three different SFTSV strains. This assay exhibited comparable sensitivity to qRT-PCR and 10-fold more sensitivity than conventional RT-PCR, with a rapid detection time of 30 to 60 minutes. The RT-LAMP assay using SFTSV clinical specimens has demonstrated a similar detection rate to qRT-PCR and a higher detection rate compared to conventional RT-PCR. Moreover, there was no observed cross-reactive amplification of other human infectious viruses including Japanese Encephalitis Virus (JEV), Dengue, Enterovirus, Zika, Influenza and Middle East Respiratory Syndrome Coronavirus (MERS-CoV). This highly sensitive, electro- and equipment-free rapid colorimetric visualization method is feasible for resource-limited SFTSV field diagnosis.

Keywords: SFTSV, RT-LAMP, colorimetric visualization, pocket warmer

\section{Introduction}

In 2009, severe fever with thrombocytopenia syndrome virus (SFTSV), a novel virus belonging to the family of Bunyaviridae, emerged as an infectious disease in China [1]. From 2010 to 2016, humans infected with SFTSV in China, Japan and South Korea had a reported average case fatality rate of 5.3 32\% (www.nih.go.jp/niid/en/basic-science/ $865 \ldots / 6339$-tpc433) [2, 3]. Studies have reported that about
$10 \%$ of SFTSV infections are due to bites of Haemaphysalis longicornis, the implicated vector of this viral infection [4, 5]. Moreover, the recent reports of human-to-human SFTSV transmission in China and Korea have raised public health concerns [6-8]. The main symptoms observed in patients infected with SFTSV include high fever, thrombocytopenia, leukocytopenia, gastrointestinal symptoms, and to some extent hemorrhages and multi-organ dysfunction symptoms $[1,9,10]$. However, these SFTSV-associated clinical symptoms 
are nonspecific as they may also be observed in other infectious diseases $[1,11]$. Therefore, as an adjunct to these clinical symptoms, rapid and accurate diagnosis is highly essential to confirm SFTSV infection. A reliable diagnosis is crucial to determine the appropriate treatment and increase the survival rate of infected patients [12]. To the present, diagnosis of SFTSV infection utilizes methods such as enzyme-linked immunosorbent assays (ELISA) [13], virus isolation, and quantitative reverse transcription-polymerase chain reaction (qRT-PCR) [12]. However, these detection methods may have limitations such as low-accuracy, timeconsuming nature, need for well-trained technicians and/ or expensive equipment.

Recently, a nucleic acid amplification test (NAAT)-based method called loop-mediated isothermal amplification combined with reverse transcription (RT-LAMP) has been developed for the diagnosis of a broad-spectrum RNA viral pathogen [14, 15]. The method demonstrated high specificity, efficiency and sensitivity similar to conventional RT-PCR based detection methods. A significant amount of target DNA or RNA can be amplified within a span of 30 to $60 \mathrm{~min}$ at a constant temperature of $60-65^{\circ} \mathrm{C}[16,17]$. In this study, an RT-LAMP system was developed for the diagnosis of the SFTSV infection whose diagnostic results could be observed and confirmed by the one-pot based colorimetric visualization system. Innovatively, this study was also to develop a reaction platform that would facilitate an electro- and equipment-free method of diagnosis. Thus, this can surpass the currently available methods with its advantages of rapid, simplified, low-cost and comparable sensitivity [18]. Furthermore, this diagnostic technique has the potential advantage of being used in resource-limited primary care hospitals in rural areas, where SFTSV prevalently causes human infection. This assay can also be applied for the detection of SFTSV vector's intermediate hosts, the livestock animals, to prevent the occurrence of human infection $[19,20]$.

\section{Materials and Methods}

\section{Virus, Titration and RNA Extraction}

Three SFTSV strains (CB1/2014, CB2/2015, and CB3/2016) were isolated from serum samples collected from the Chungbuk National University Hospital, South Korea in accordance with the approved procedures by the Institutional Review Board of Chungbuk National University Hospital (IRB No. 2017-05-002001). The SFTS viruses were propagated and titrated using the foci forming unit (FFU) method [21] in Vero E6 cells. In vitro experiments using SFTSV were conducted in an Enhanced Bio Safety Level 3 (BL-3+) facility at Chungbuk National University approved by Korea Centers for Disease Control and Prevention. RNA was extracted from $200 \mu$ l of virus culture supernatant or patient serum samples using RNeasy Mini Kit (QIAGEN, China) in accordance to the manufacturer's protocol. The RNA was eluted into $50 \mu \mathrm{l}$ of RNase-free distilled water and immediately used or stored at $-80^{\circ} \mathrm{C}$.

\section{Design of RT-LAMP Primers}

A total of 154 genomic sequences of different strains of SFTSV were retrieved from GenBank (https://www.ncbi.nlm.nih.gov/ genbank/) and analyzed using the CLC Main workbench 7 (version 7.6.4.) to identify conserved regions of the nucleotide sequence of SFTSV. Two sets of six SFTSV-specific RT-LAMP primers targeting $\mathrm{M}$ and $\mathrm{L}$ genes were designed using the Primer Explorer V4 software program (http://primerexplorer.jp/ elamp4.0.0/index.html) and consist of two external primers (forward outer primer F3 and backward outer primer B3), two internal primers (forward inner primer FIP and backward inner primer BIP) and two loop primers (backward loop primer LB and forward loop primer LF). The details of the primer set selected and used in this study are shown in Table 1 while the overall schematic primer design is depicted in Fig. 1.

Optimization of RT-LAMP Conditions and Analysis of the RTLAMP Product

The RT-LAMP reaction was performed in a final reaction volume of $10 \mu \mathrm{l}$ using the Warm Start Colorimetric LAMP 2x Master Mix

Table 1. RT-LAMP primer sequences in L gene of SFTSV.

\begin{tabular}{llll}
\hline \multicolumn{1}{c}{ Primer } & \multicolumn{1}{c}{ Sequence $\left(5^{\prime} \rightarrow 3^{\prime}\right)$} & Position \\
\hline RT-LAMP & F3 & TTCCCTCAGGCTCAAGAGT & $4393-4411$ \\
& B3 & AGACGGGGTCCAAGCTTAG & $4580-4598$ \\
& FIP (F2-F1C) & GACAATGTTTCTCTCCCTAA-TGCACATTGCTTGAGGAAGT & $4469-4488,4423-4442$ \\
& BIP (B2-B1C) & TTCCAAGAGCCAGTGGACTTGC-CCAAACCACACCTCTGACAC & $4507-4528,4549-4568$ \\
& LF & CAACAAATTTCCCTGTTAGGTGTTC & $4444-4468$ \\
& LB & GGTGCAAGGCAGAAGATCTG & $4529-4548$ \\
& FCR & TAGACATCAAGACCGACTCA & $1031-1050$ \\
& Reverse & TGACTACCCCCTCCACATT & $1126-1144$ \\
\hline
\end{tabular}




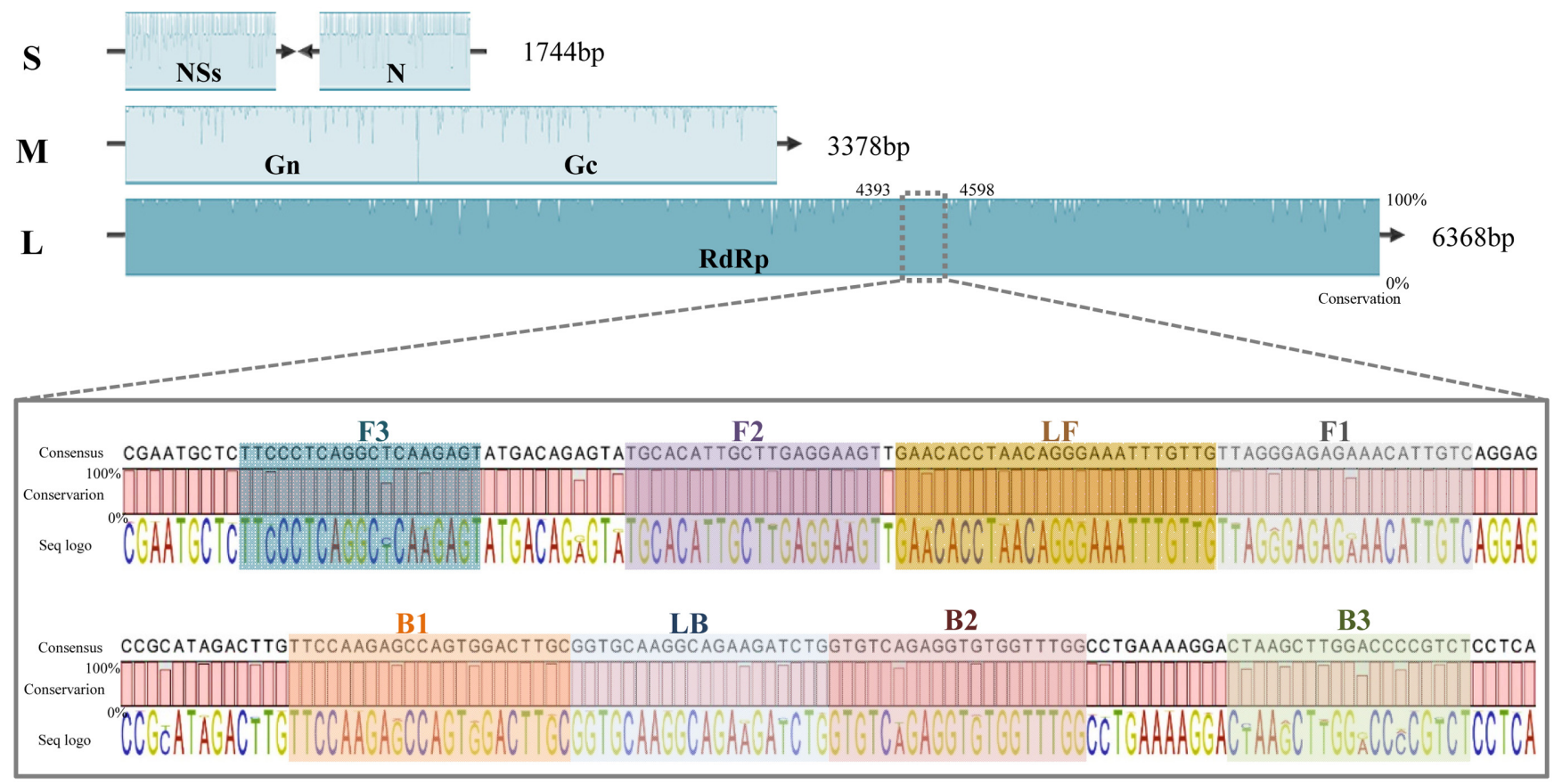

Fig. 1. Alignment of SFTSV sequences for RT-LAMP primer design.

The highly-conserved region in the L segment of 154 SFTSV sequences was obtained using the CLC Main workbench 7 (version 7.6.4.). FIP (Forward Inner Primer) contains F2 and F1c (F1 complementary sequence), and BIP (Backward Inner Primer) contains B2 and B1c (B1 complementary sequence). The forward and backward loop primers (LF and LB, respectively) were additionally designed to enhance the sensitivity of the assay. Please see Table 1 for more details of the SFTSV RT-LAMP primer sequences.

(New England Biolabs Inc, USA). The RT-LAMP mixture contained $2 \mu \mathrm{l}$ of RNA, $1 \mu \mathrm{l}$ outer primer (F3, B3; $5 \mu \mathrm{M}), 1 \mu \mathrm{l}$ inner primer (FIP, BIP; $80 \mu \mathrm{M}$ ), $1 \mu \mathrm{l}$ loop primer (LF, LB; $20 \mu \mathrm{M}$ ), and $5 \mu \mathrm{l} 2 \mathrm{x}$ Master Mix. The positive reaction of the RT-LAMP was visually determined with a color change from pink to yellow, which is a result of nucleic acid amplification. To verify the optimal and earliest reaction time for positive color change discriminable by naked eye, RT-LAMP reactions were performed at $65^{\circ} \mathrm{C}$ and the results were recorded between 10 to $40 \mathrm{~min}$ in $10 \mathrm{~min}$ intervals. In addition, the RT-LAMP products were electrophoresed on $2 \%$ agarose gel in 1\% Tris-acetate-EDTA (TAE) buffer. The amplified products were confirmed by the specific ladder-like pattern on a UV illuminator and documented using a gel documentation system (Gel Doc XR Plus, Bio-Rad, USA).

\section{Sensitivity Evaluation of RT-LAMP Assay}

RNA samples were serially diluted 10-fold and used for comparative sensitivity experiments. The RT-LAMP reaction was carried out as described above. The qRT-PCR reaction was performed using the SFTSV specific RT-PCR primers (forward and reverse) (Table 1) and the TOPreal One-step RT qPCR Kit (SYBR Green, low Rox) (Enzynomics, Korea) under the following conditions: reverse transcription at $42^{\circ} \mathrm{C}$ for $30 \mathrm{~min}$, initial denaturation at $95^{\circ} \mathrm{C}$ for $10 \mathrm{~min}, 35$ cycles of three steps (denaturation at $95^{\circ} \mathrm{C}$ for $30 \mathrm{sec}$, annealing at $60^{\circ} \mathrm{C}$ for $30 \mathrm{sec}$, elongation at $72^{\circ} \mathrm{C}$ for $30 \mathrm{sec}$ ), and final elongation at $72^{\circ} \mathrm{C}$ for $5 \mathrm{~min}$. Also, conventional RT-PCR reactions were performed using the same primers as above and the TOPscript One-step RT PCR Kit (Enzynomics, Korea) under the same conditions conducted for qRT-PCR described above.

\section{Specificity of SFTSV in Clinical Samples}

RNA extracted from serum samples collected from patients admitted in Chungbuk National University Hospital with symptoms of high fever was used for RT-LAMP, conventional RTPCR and qRT-PCR assays. To evaluate the specificity of the established SFTSV RT-LAMP assay, cross-reactivity tests were performed using RNA from: (i) Japanese Encephalitis Virus (JEV), (ii) Dengue Fever Virus (serotype 1, 2, 3, and 4), (iii) Enteroviruses [EV71 (41401/KCDC/2011), E6 (Korea/CHP3/2015), E18 (Korea/ CHP131/2016), and B5 (Korea/ CHP133/2016)], (iv) Zika virus (ATCC/VR-1859), (v) Influenza virus [H1N1 (A/California/04/ 2009), H3N2 (A/Perth/16/2009), and type B (B/Brisbane/60/ 2008) ] and (vi) MERS-CoV (KT02139/Korea/2015).The presence of RNA from these viruses used for the cross-reactivity evaluation was confirmed using virus-specific primers (Table 2).

\section{Establishment of Electro-Free Reaction Platform}

The electro-free RT-LAMP reaction platform utilizes a pocket warmer to perform the optimized RT-LAMP reaction for SFTSV detection. To compare the results of RT-LAMP assays performed 
Table 2. RT-PCR primers for specific amplification of other human infectious viruses.

\begin{tabular}{lll}
\hline \multicolumn{1}{c}{ Virus } & $\begin{array}{c}\text { Primer } \\
\text { name }\end{array}$ & \multicolumn{1}{c}{ Sequence $\left(5^{\prime} \rightarrow 3^{\prime}\right)$} \\
\hline JEV & JEV-F & GGGGACAAGCAGATTAACCA \\
& JEV-R & CCCCAAAGAGCGTTCTGAA \\
Dengue* $^{*}$ & D1 & TCAATATGCTGAAACGCGCGAGAAACCG \\
& D2 & TTGCACCAACAGTCAATGTCTTCAGGTTC \\
Entero & EV71-F & TGATATCCTGCAGACGGGCA \\
EV71 & EV71-R & TGCACGCAACRAAAGTGAACT \\
Entero & E6-F & CCHGCDCTHACCGCWGTGGARACDGG \\
E6 & E6-R & GGRSCNCCDGGWGGYACAWACAT \\
Entero & E18-F & CCHGCDCTHACCGCWGTGGARACDGG \\
E18 & E18-R & GGRSCNCCDGGWGGYACAWACAT \\
Entero & B5-F & CCHGCDCTHACCGCWGTGGARACDGG \\
B5 & B5-R & GGRSCNCCDGGWGGYACAWACAT \\
Zika & Zika-F & TTCACCAAGAGCCGAAGC \\
& Zika-R & TCCTTGAACTCTACCAGTGCTT \\
H1N1 & H1N1-F & AGCAAGAAGTTCAAGCCG \\
& H1N1-R & CGTGAACTGGTGTATCTGAA \\
H3N2 & H3N2-F & GGGGTTACTTCAAAATACGAAG \\
& H3N2-R & GTTGCCAATTCAGAGTGTT \\
B & B-F & CAGGAAGAGTAAAACATACTGAGGA \\
& B-R & GATTCGCAAGGCCCTGTT \\
MERS- & MERS-F & GGAATGGAATTAAGCAACTGGC \\
CoV & MERS-F & CGCGAATTGTGAACAATAGCT \\
\hline
\end{tabular}

*Sequence of dengue primers are obtained from Lanciotti et al. [27]

in a PCR machine over this reaction platform, parallel testing was conducted. The heating reaction in the pocket warmer was initiated by shaking it several times. To monitor the temperature at the initial and during the reaction time, the study utilized a temperature-indicating sticker (Artmagics of Korea), that changes in color (black, pink or red) to indicate temperatures from $50^{\circ} \mathrm{C}$ to $70^{\circ} \mathrm{C}$ with $5^{\circ} \mathrm{C}$ intervals, attached on the surface of the hand warmer. The reaction tubes were placed between two hand warmers in a pocket once the inside of the stacked hand warmer had reached a temperature of $65^{\circ} \mathrm{C}$ (Fig. 5). The reaction tubes were incubated in the pocket warmers for a total of $60 \mathrm{~min}$ and the results were analyzed by colorimetric visualization as performed in the RT-LAMP assay using a PCR machine.

\section{Results}

\section{Optimizing the Efficiency and Speed of an RT-LAMP} Assay for the Detection of SFTSV

From the total of 154 genomic sequences of SFTSV retrieved from GenBank with identified highly-conserved regions in $\mathrm{M}$ and $\mathrm{L}$ gene segments, the most efficient primer set designed was selected from the sets of primer candidates for the RT-LAMP assay. From the various range of concentrations evaluated [from 2.5 to $20 \mu \mathrm{M}$ for F3 and B3, from 20 to $80 \mu \mathrm{M}$ for FIP and BIP, and from 5 to $20 \mu \mathrm{M}$ for LF and LB], the optimal primer set targeting the highlyconserved region of $\mathrm{L}$ segment was selected. The details of the selected primer set and the positions of the primers in the viral genomic sequence are shown in Fig. 1 and Table 1.

The RT-LAMP reactions performed using the highest concentration of RNA extracted from 3 genetically and periodically diverse viruses $\left(10^{5}, 10^{4}\right.$, and $10^{2} \mathrm{FFU}$ of CB1/ 2014, CB2/2015, and CB3/2016, respectively) in the colorimetric RT-LAMP mixture incubated at $65^{\circ} \mathrm{C}$. The colorimetric RT-lamp reactions indicated through color change from pink (negative) to yellow (positive) can be directly visualized and interpreted by the naked eye. The reaction times were recorded for $40 \mathrm{~min}$ in $10 \mathrm{~min}$ intervals (Fig. 2A). Results have shown that within the short span of a 30-min period, positive reactions were observed when viral RNA copies are more than $10^{5}$ per reaction (CB1/2014). Furthermore, the typical amplicon pattern of RT-LAMP reactions of all SFTSV was confirmed by gel electrophoresis within $20 \mathrm{~min}$ (Fig. 2A).

\section{Sensitivity Evaluation of the RT-LAMP Assay}

The sensitivity of the RT-LAMP assay was compared to the conventional one-step RT-PCR and qRT-PCR assays for SFTSV detection by performing end-point detection using 10-fold serially diluted RNA extracted from the viruses as templates (Figs. 2B-2E). The RT-LAMP reaction was capable of detecting $10^{\circ}$ viral genome copies for all three SFTSV within $1 \mathrm{~h}$ (Fig. 2B); this is 10 times more sensitive for all viruses tested than one-step RT-PCR (which takes $150 \mathrm{~min}$ ). The qRT-PCR reaction detected up to $10^{0}$ to $10^{-1}$ viral genome copies within $120 \mathrm{~min}$, which is similar to or more sensitive than RT-LAMP (Fig. 2E). Although RT-LAMP exhibited 10-fold less sensitivity than qRT-PCR in CB2/ 2015 virus, it is worth considering the benefits of RT-LAMP over qRT-PCR, in terms of reaction rate, cost efficiency and ease of implementation. These results demonstrate that the RT-LAMP assay designed to detect SFTSV is highly capable of generating a diagnosis within a 60-min time period with comparable sensitivity to conventional RT-PCR-based methods.

\section{Specificity and Sensitivity Evaluation of SFTSV RT-LAMP Assay Using Clinical Specimens}

Twelve serum samples collected from patients exhibiting symptoms of high fever with thrombocytopenia were evaluated for SFTSV utilizing the RT-LAMP assay. With 


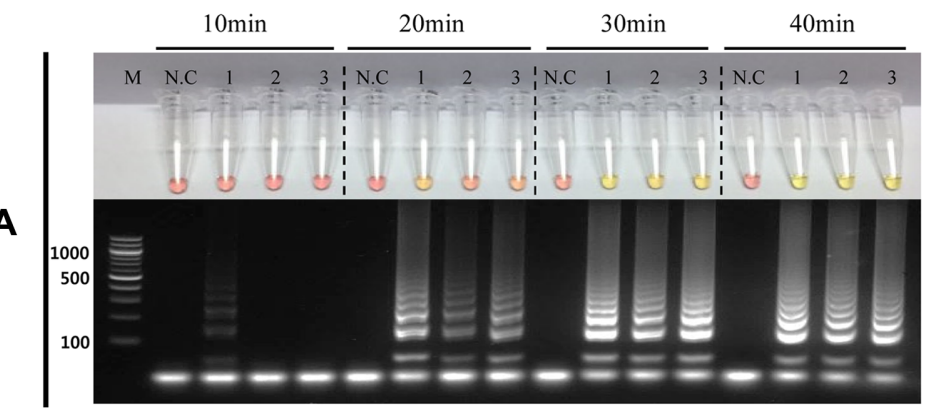

CB1/2014
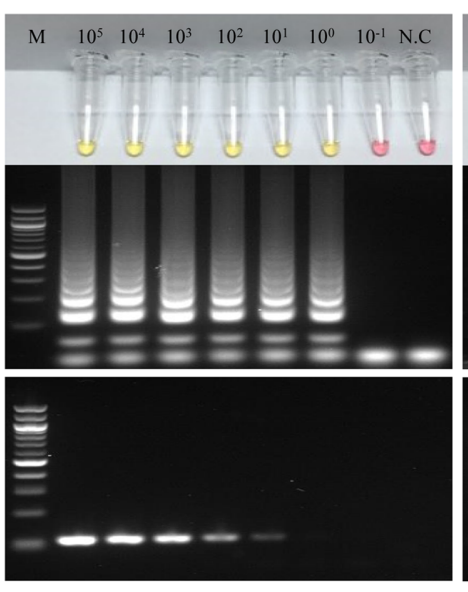

D

$\begin{array}{lllllllll}\text { Ct } & 14.8 & 18.1 & 21.9 & 25.4 & 28.6 & 32.1 & \text { N.D } & \text { N.D }\end{array}$
CB2/2015
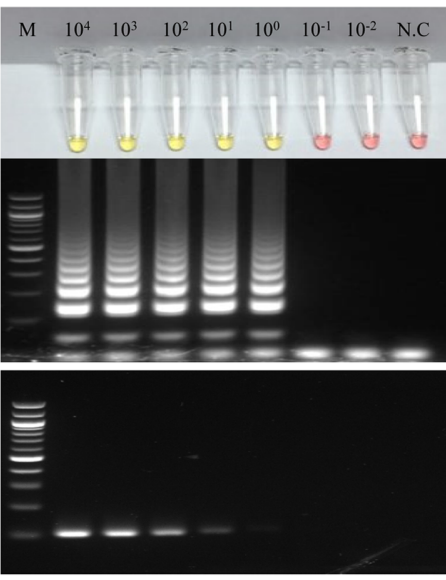

$\begin{array}{llllllll}\text { Ct } & 15.8 & 19.0 & 22.7 & 26.4 & 29.5 & 33.1 & \text { N.D N.D }\end{array}$
CB3/2016
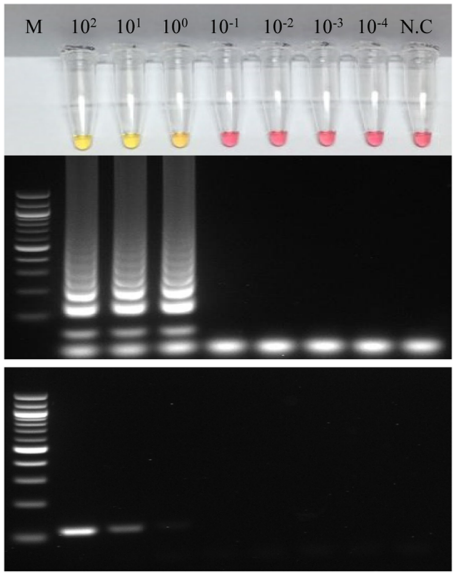

Ct 24.227 .431 .5 N.D N.D N.D N.D N.D
E

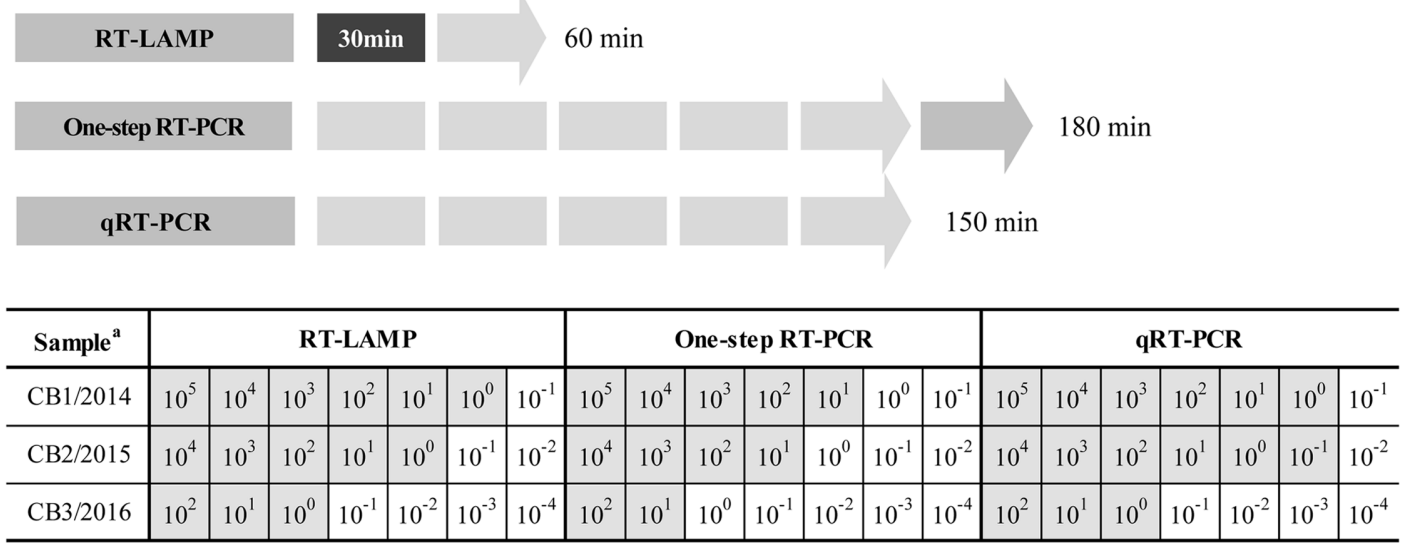

a) RNA copies (1 $\mathrm{x}) /$ Reaction

Fig. 2. Comparative sensitivity of RT-LAMP, conventional RT-PCR and qRT-PCR.

(A) Detection time observation of the visible positive reaction in 10-min intervals of the colorimetric RT-LAMP assay for the three SFTSV-positive samples. The observed color change from pink to yellow in the tube indicates a positive reaction for SFTSV. The upper panel shows the colorimetric visualization of the RT-LAMP reaction and the lower panel shows the image of agarose gel electrophoresis of the RT-LAMP reaction (M, 100 bp ladder size marker; N.C, negative control; Lane 1, CB1/2014; Lane 2, CB2/2015; Lane 3, CB3/2016). RNA samples extracted from three SFTSVs $\left(10^{5}, 10^{4}\right.$, and $10^{2}$ infectious viral particles per reaction for CB1/2014, CB2/2015, and CB3/2016, respectively) were 10-fold serially diluted and used for determining the sensitivity of (B) RT-LAMP in comparison with (C) the conventional RT-PCR and (D) qRT-PCR. The conventional RT-PCR products were confirmed by agarose gel electrophoresis and the positive reaction of qRT-PCR amplification was represented by $\mathrm{Ct}$ values in each RNA dilution point. (E) Summary of the approximate processing time and sensitivity of the SFTSV RT-LAMP assay, conventional RT-PCR and qRT-PCR. The processing time required for each experiment is represented by an arrow graph indicating 30 min per block. Grey-highlighted cells in the table represent the dilution points that conferred positive reactions in each of the three diagnostic methods. N/A: not available. 


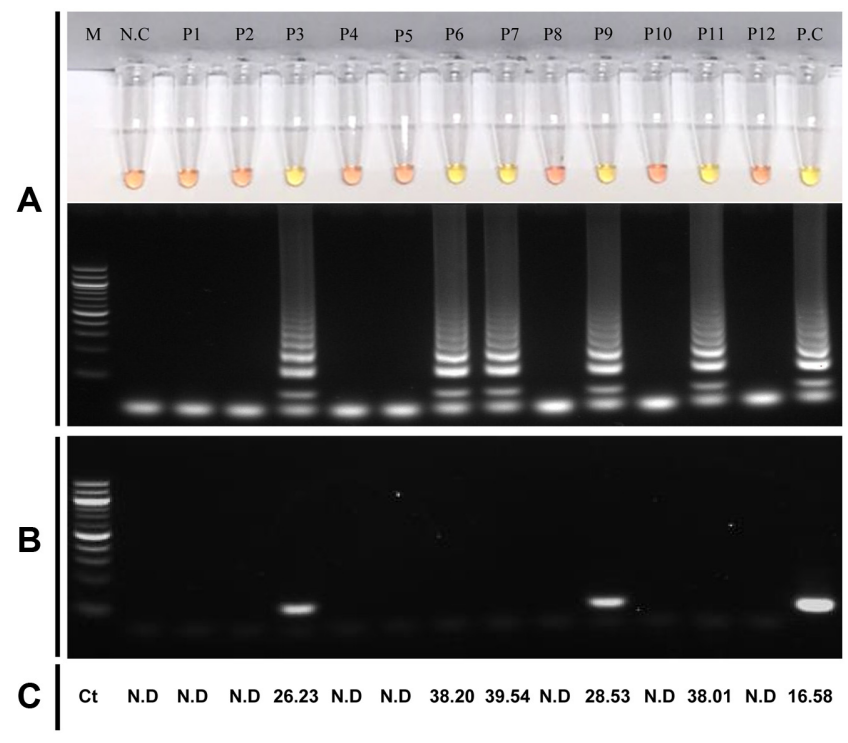

Fig. 3. Clinical evaluation of the RT-LAMP assay for SFTSV detection.

A total of 12 serum samples collected from patients suspected of SFTSV infection was used to perform (A) the direct RT-LAMP assay, (B) one-step RT-PCR, and (C) qRT-PCR. The positive color change (pink to yellow) of direct RT-LAMP can be visually observed by naked-eye and can be confirmed using agarose gel electrophoresis. The positive results in one-step RT-PCR and qRT-PCR were presented by positive band in agarose gel electrophoresis and $\mathrm{Ct}$ value, respectively. $\mathrm{M}$ : 100 bp DNA ladder; P.C: positive control; N.D: not detected.

the same set of RNA extracted from the serum samples, a consequent parallel testing was also conducted using conventional one-step RT-PCR and qRT-PCR were also conducted for confirmation and comparison of the positive result. Out of the 12 samples, 5 positive samples were identified using the RT-LAMP assay (Fig. 3A). The direct RT-LAMP and qRT-PCR equally diagnosed the SFTSV from 5 clinical samples. However, the one-step RT-PCR has only detected 2 out of 5 SFTSV positive samples from the previously mentioned methods (Figs. 3B and 3C). The qRTPCR products of SFTSV-positive clinical samples were further confirmed through sequencing. The results suggest that the developed RT-LAMP assay using clinical samples has not only improved the accuracy and sensitivity of this NAAT-based detection method but also increases its feasibility as a point-of-care test (POCT).

\section{The Specificity of RT-LAMP Assay Using Other Human Infectious Viruses}

The specificity of the SFTSV RT-LAMP assay was also tested using RNAs of other human infectious viruses that can also cause symptoms of high fever like the SFTSV, which includes JEV, Dengue serotype 1 to 4, EV71, Echo 6, E18, Coxsackie B5, Zika, and human respiratory viruses including influenza H1N1, H3N2, type B, and MERS-CoV (Fig. 4). Results have shown that RT-LAMP assay was negative for all human infectious viruses tested except for SFTSV which yielded a positive result (Figs. 4A and 4B). Thus, the RT-LAMP assay demonstrated high clinical application for SFTSV detection which has similar sensitivity to qRTPCR and with no observed cross-reaction with the other human infectious viruses tested.

\section{Use of Alternative RT-LAMP Reaction Platform}

Because SFTSV human infection mostly occurs in rural areas where expensive diagnostic equipment is often lacking, in this study, we used a cheap and commercially available alternative reaction platform for the SFTSV RTLAMP assay, the pocket warmers. In addition, this study also makes use of temperature-indicating stickers, attached on the pocket warmer's surface, to easily monitor the optimal temperature needed for this RT-LAMP reaction $\left(65^{\circ} \mathrm{C}\right)$ (Fig. 5A). Using the end-point dilution of the three SFTSV RNA samples to verify the sensitivity of the established platform, parallel testing of the pocket warmer and PCR machine was performed. Results show similar detection sensitivity observed in the diluted samples performed in both reaction platforms indicating comparable detection performance (Fig. 5B). The results also suggest that the commercially available pocket warmer can be used as an alternative platform to perform the developed RTLAMP assay for SFTSV detection, facilitating a nearly electro- and equipment-free diagnosis in a resource-limited field setting.

\section{Discussion}

Human infections with SFTSV have most often occurred in rural areas partly because ticks, which are the known vectors or transmitter of the virus, generally thrive in areas where their hosts such as livestock and wild animals thrive [22]. These areas often lack facilities with proper clinical and laboratory equipment to accurately diagnose SFTSV infections. In this study, we developed a simple and rapid molecular diagnostic RT-LAMP method for SFTSV infection which is able to detect approximately 1 copy of viral genome comparable to qRT-PCR. For more widespread application, the RT-LAMP detection system is combined with one-pot colorimetric visualization and performed using an alternative reaction platform such as the commercially available pocket 

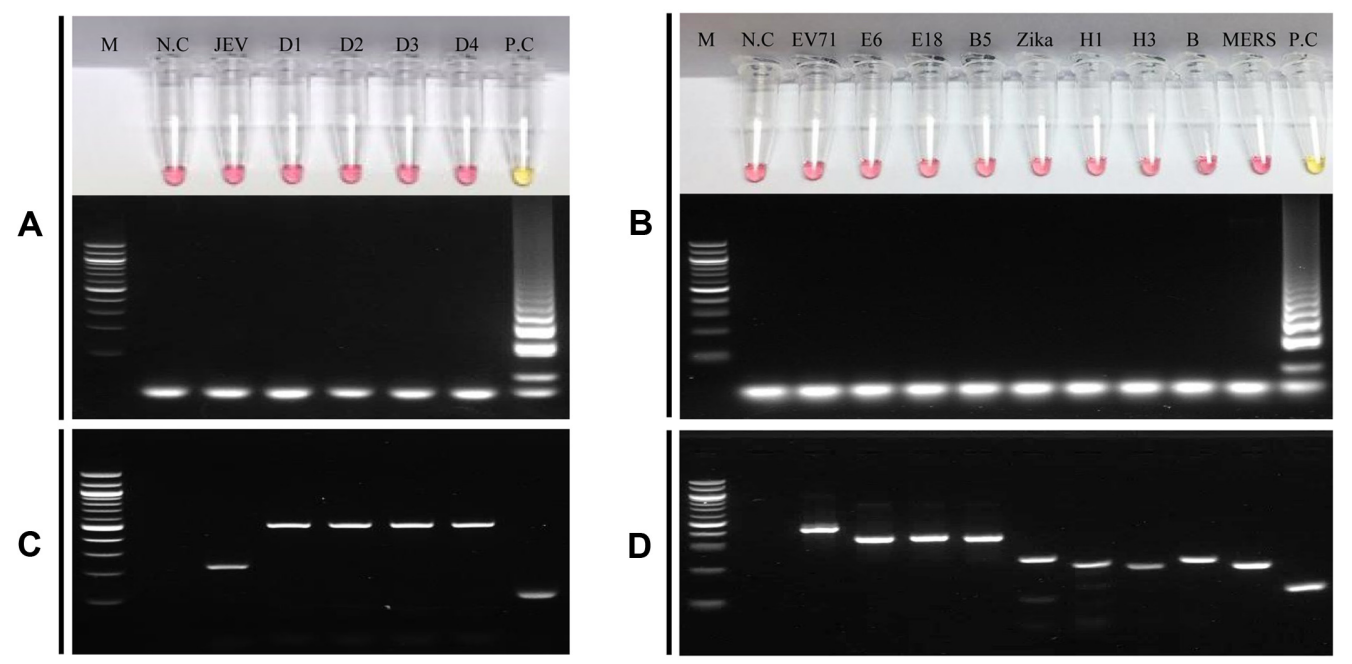

Fig. 4. Specificity of SFTSV RT-LAMP assay in comparison with one-step RT-PCR and qRT-PCR for SFTSV and other human infectious viruses.

Various human infectious viruses (JEV, Japanese Encephalitis Virus; D1, Dengue Fever Virus serotype 1; D2, Dengue Fever Virus serotype 2; D3, Dengue Fever Virus serotype 3; D4, Dengue Fever Virus serotype 4; EV71, Enterovirus A71; E6, EchovirusE-6; E18, EchovirusE-18; B5, Coxsackie virus B5; Zika, Zika virus; H1, Influenza virus H1N1; H3, Influenza virus H3N2; B, Influenza virus type B; MERS, MERS-CoV) were used to verify the specificity of the SFTSV RT-LAMP assay (A and B) and the presence of the RNA extracted from each human infectious virus was confirmed by one-step RT-PCR assay (C and D). M: 100 bp DNA ladder; N.C: negative control; P.C: positive control.

warmer, facilitating a mobile, electro- and equipment-free set up for diagnosis of SFTSV infection.

Recently, although similar RT-LAMP assays for the detection of SFTSV have been developed [18, 23, 24], the need for expensive immobile electric equipment for reaction or visualization in performing the RT-LAMP assay limits its use for field diagnosis especially in a resourcelimited setting. In this study, the colorimetric visualization system enables the one-pot observation of results without any additional steps (e.g., adding fluorescence dye or running the product in gel electrophoresis) or visualizing equipment (e.g., UV illuminator) to observe the result of the reaction. Unlike the methods utilizing expensive PCR or qPCR machines, RT-LAMP assay minimally requires the use of lower-cost equipment such as electrically powered heat block or water bath that can provide a consistent temperature between $60^{\circ} \mathrm{C}$ to $65^{\circ} \mathrm{C}$ for 40 to $60 \mathrm{~min}$. However, in this study we were able to innovatively utilize a pocket warmer system in performing the RT-LAMP assay, which can be easily carried out in the field as previously demonstrated and designed in recent related studies in diagnosing influenza virus and Anthrax, also using the pocket warmer for the RT-LAMP assay [16, 17, 25]. To verify that the temperature of the pocket warmer is optimal and constantly maintained, the test was carried out using a color-changing temperature indicating sticker which is cheap and reusable instead of using a thermometer. Therefore, this system can be adapted and used in rural areas and developing countries where the availability of expensive equipment is limited. Furthermore, the single use of the disposable pocket warmers could be advantageous in minimizing cross-contamination among different batches of samples.

The RNA extraction step can be a limitation for the RTLAMP to be genuinely used as a point-of-care test (POCT). Interestingly, RT-LAMP assay was successfully performed without the RNA sample preparation step in case of some RNA viruses, which reduces the sample processing time and cost, increasing its feasibility as a diagnostic method for POCT [26]. Our RT-LAMP was also able to detect SFTSV directly using viral particles diluted in human serum without viral RNA extraction in $10 \mathrm{mM}$ Tris-HCl buffer ( $\mathrm{pH}$ 8.0, 1:10 dilution) (data not shown). The temperature condition of the RT-LAMP reaction $\left(65^{\circ} \mathrm{C}\right)$ used in this study is possibly high enough to directly detect and amplify the genome of SFTSV particles in serum samples but decreased sensitivity occurred due to the additional dilution $(1: 10)$ of serum samples in the Tris- $\mathrm{HCl}$ buffer. On the other hand, the development of a simple RNA extraction method using a one-step syringe filter system to purify and concentrate the viral genomes could be ideal for RT-LAMP reaction as a POCT. 


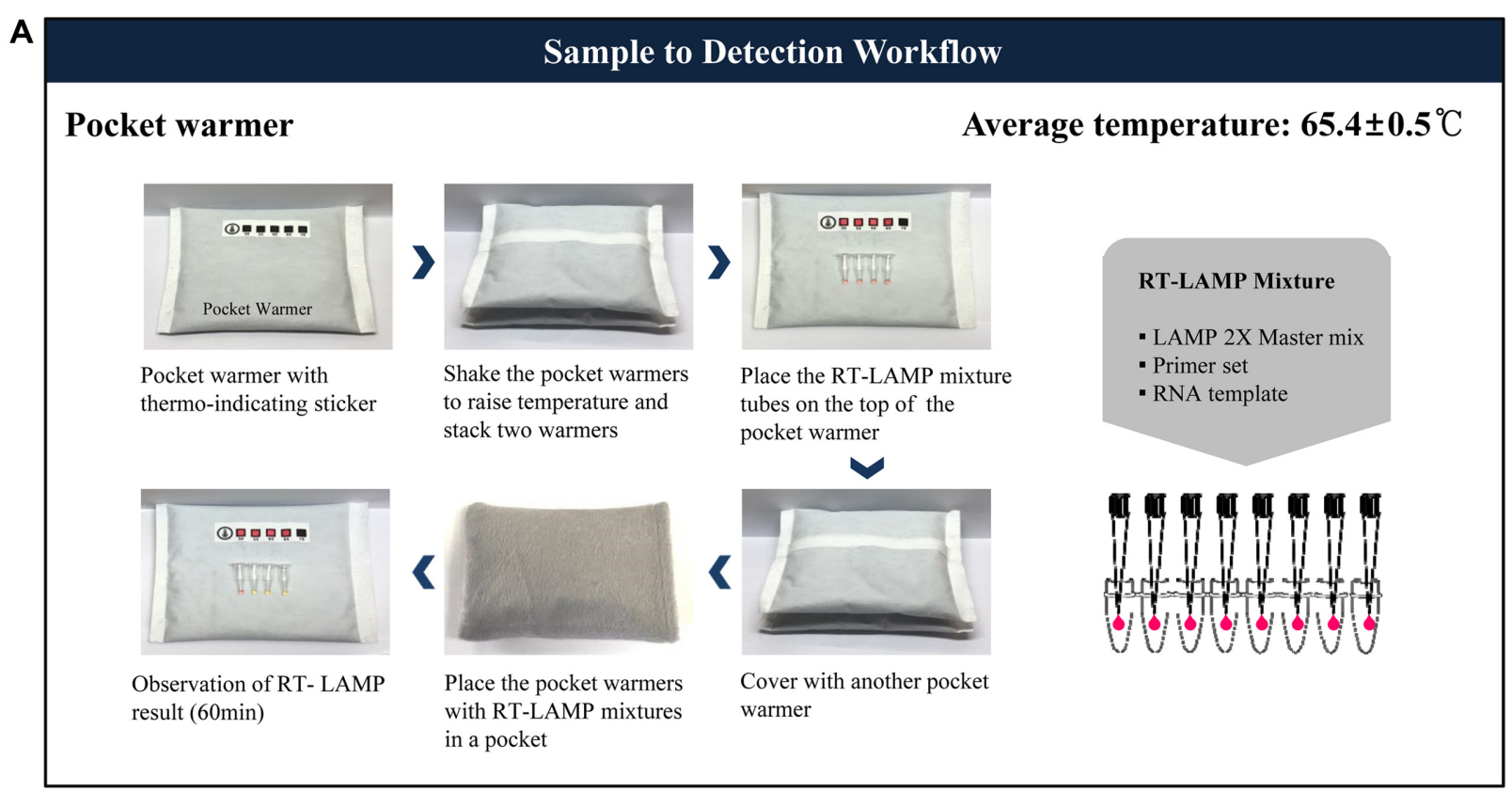

\begin{tabular}{|c|c|c|c|c|c|c|c|c|c|c|c|c|c|c|}
\hline \multirow{3}{*}{$\begin{array}{c}\text { Sample }^{a} \\
\text { CB1/2014 }\end{array}$} & \multicolumn{14}{|c|}{ RT-LAMP } \\
\hline & \multicolumn{7}{|c|}{ PCR machine } & \multicolumn{7}{|c|}{ Pocket warmer } \\
\hline & $10^{5}$ & $10^{4}$ & $10^{3}$ & $10^{2}$ & $10^{1}$ & $10^{0}$ & $10^{-1}$ & $10^{5}$ & $10^{4}$ & $10^{3}$ & $10^{2}$ & $10^{1}$ & $10^{0}$ & $10^{-1}$ \\
\hline CB2/2015 & $10^{4}$ & $10^{3}$ & $10^{2}$ & $10^{1}$ & $10^{0}$ & $10^{-1}$ & $10^{-2}$ & $10^{4}$ & $10^{3}$ & $10^{2}$ & $10^{1}$ & $10^{0}$ & $10^{-1}$ & $10^{-2}$ \\
\hline CB3/2016 & $10^{2}$ & $10^{1}$ & $10^{0}$ & $10^{-1}$ & $10^{-2}$ & $10^{-3}$ & $10^{-4}$ & $10^{2}$ & $10^{1}$ & $10^{0}$ & $10^{-1}$ & $10^{-2}$ & $10^{-3}$ & $10^{-4}$ \\
\hline
\end{tabular}

a) RNA copies $(1 \mathrm{x}) /$ Reaction

Fig. 5. Schematic workflow and comparative evaluation of an alternative RT-LAMP reaction platform for SFTSV detection. (A) Schematics of sample preparation and experimental procedures of using pocket warmer as a detection method. The average temperature of the pocket warmer during the reaction time is also shown. (B) Comparative RT-LAMP reaction was performed using the end-point dilution of the three SFTSV RNA samples in both PCR machine and pocket warmer platform. Grey-highlighted cells in the table represent the dilution points that conferred positive reactions in both reaction platforms.

Our direct RT-LAMP system combined with one-pot colorimetric visualization and alternative pocket warmer reaction platform for detection of SFTSV in this study provides a simple, fast and feasible method for early, such as acute phase, clinical diagnosis of SFTSV infection. Overall, this system can highly improve and contribute to efficient SFTSV diagnosis and relevant epidemiological surveillance.

\section{Conflict of Interest}

The authors have no financial conflicts of interest to declare.

\section{Acknowledgments}

This work was supported by the Basic Science Research Program of the Korea Health Technology R\&D Project through the Korea Health Industry Development Institute (KHIDI), funded by the Ministry of Health \& Welfare, Republic of Korea [grant number: HI15C2888 to M.-S.S and HI15C2817 to Y.K.C] and by a research grant from Chungbuk National University in 2014 to M.-S.S.

\section{References}

1. Yu X-J, Liang M-F, Zhang S-Y, Liu Y, Li J-D, Sun Y-L, et al. 
2011. Fever with thrombocytopenia associated with a novel bunyavirus in China. N Engl. J. Me 364: 1523-1532.

2. Zhan J, Wang Q, Cheng J, Hu B, Li J, Zhan F, et al. 2017. Current status of severe fever with thrombocytopenia syndrome in China. Virol. Sin. 32: 51-62.

3. Park S-W, Ryou J, Choi W-Y, Han M-G, Lee W-J. 2016. Epidemiological and clinical features of severe fever with thrombocytopenia syndrome during an outbreak in South Korea, 2013-2015. Am. J. Trop. Med. Hyg. 95: 1358-1361.

4. Niu G, Li J, Liang M, Jiang X, Jiang M, Yin H, et al. 2013. Severe fever with thrombocytopenia syndrome virus among domesticated animals, China. Emerg. Infect. Dis. 19: 756.

5. Zhang Y-Z, Xu J. 2016. The emergence and cross species transmission of newly discovered tick-borne Bunyavirus in China. Curr. Opin. Virol. 16: 126-131.

6. Kim WY, Choi W, Park S-W, Wang EB, Lee W-J, Jee Y, et al. 2015. Nosocomial transmission of severe fever with thrombocytopenia syndrome in Korea. Clin. Infect. Dis. 60: 1681-1683.

7. Liu Y, Li Q, Hu W, Wu J, Wang Y, Mei L, et al. 2012. Personto-person transmission of severe fever with thrombocytopenia syndrome virus. Vector Borne Zoonotic Dis. 12: 156-160.

8. Bao C-j, Guo X-1, Qi X, Hu J-l, Zhou M-h, Varma JK, et al. 2011. A family cluster of infections by a newly recognized bunyavirus in eastern China, 2007: further evidence of personto-person transmission. Clin. Infect. Dis. 53: 1208-1214.

9. Li S, Xue C, Fu Y, Wang J, Ding X, Liu R, et al. 2011. Sporadic case infected by severe fever with thrombocytopenia syndrome bunyavirus in a non-epidemic region of China. Biosci. Trends 5: 273-276.

10. Xu B, Liu L, Huang X, Ma H, Zhang Y, Du Y, et al. 2011. Metagenomic analysis of fever, thrombocytopenia and leukopenia syndrome (FTLS) in Henan Province, China: discovery of a new bunyavirus. PLoS Pathog. 7: e1002369.

11. Zhang Y-Z, Zou Y, Fu ZF, Plyusnin A. 2010. Hantavirus infections in humans and animals, China. Emerg. Infect. Dis. 16: 1195.

12. Sun Y, Liang M, Qu J, Jin C, Zhang Q, Li J, et al. 2012. Early diagnosis of novel SFTS bunyavirus infection by quantitative real-time RT-PCR assay. J. Clin. Virol. 53: 48-53.

13. Jiao Y, Zeng X, Guo X, Qi X, Zhang X, Shi Z, et al. 2012. Preparation and evaluation of recombinant severe fever with thrombocytopenia syndrome virus nucleocapsid protein for detection of total antibodies in human and animal sera by double-antigen sandwich enzyme-linked immunosorbent assay. J. Clin. Microbiol. 50: 372-377.

14. Notomi T, Okayama H, Masubuchi H, Yonekawa T, Watanabe K, Amino N, et al. 2000. Loop-mediated isothermal amplification of DNA. Nucleic Acids Res. 28: e63-e63.
15. Fukuta S, Iida T, Mizukami Y, Ishida A, Ueda J, Kanbe M, et al. 2003. Detection of Japanese yam mosaic virus by RTLAMP. Arch. Virol. 148: 1713-1720.

16. Hatano B, Goto M, Fukumoto H, Obara T, Maki T, Suzuki G, et al. 2011. Mobile and accurate detection system for infection by the 2009 pandemic influenza A (H1N1) virus with a pocket-warmer reverse-transcriptase loop-mediated isothermal amplification. J. Med. Virol. 83: 568-573.

17. Hatano B, Maki T, Obara T, Fukumoto H, Hagisawa $K$, Matsushita Y, et al. 2010. LAMP using a disposable pocket warmer for anthrax detection, a highly mobile and reliable method for anti-bioterrorism. Jpn. J. Infect. Dis. 63: 36-40.

18. Xu H, Zhang L, Shen G, Feng C, Wang X, Yan J, et al. 2013. Establishment of a novel one-step reverse transcription loop-mediated isothermal amplification assay for rapid identification of RNA from the severe fever with thrombocytopenia syndrome virus. J. Virol. Methods 194: 21-25.

19. Kim K-H, Yi J, Kim G, Choi SJ, Jun KI, Kim N-H, et al. 2013. Severe fever with thrombocytopenia syndrome, South Korea, 2012. Emerg. Infect. Dis. 19: 1892.

20. Li Z, Hu J, Bao C, Li P, Qi X, Qin Y, et al. 2014. Seroprevalence of antibodies against SFTS virus infection in farmers and animals, Jiangsu, China. J. Clin. Virol. 60: 185-189.

21. Park J-S, Um J, Choi Y-K, Lee YS, Ju YR, Kim SY. 2016. Immunostained plaque assay for detection and titration of rabies virus infectivity. J. Virol. Methods 228: 21-25.

22. Wang S, Li J, Niu G, Wang X, Ding S, Jiang X, et al. 2015. SFTS virus in ticks in an endemic area of China. Am. J. Trop. Med. Hyg. 92: 684-689.

23. Huang X-Y, Hu X-N, Ma H, Du Y-H, Ma H-X, Kang K, et al. 2014. Detection of new bunyavirus rna by reverse transcriptionloop-mediated isothermal amplification. J. Clin. Microbiol. 2: 531-535.

24. Yang G, Li B, Liu L, Huang W, Zhang W, Liu Y. 2012. Development and evaluation of a reverse transcription loopmediated isothermal amplification assay for rapid detection of a new SFTS bunyavirus. Arch. Virol. 157: 1779-1783.

25. Ablordey A, Amissah DA, Aboagye IF, Hatano B, Yamazaki T, Sata T, et al. 2012. Detection of Mycobacterium ulcerans by the loop mediated isothermal amplification method. PLoS Negl. Trop. Dis. 6: e1590.

26. Priye A, Bird SW, Light YK, Ball CS, Negrete OA, Meagher RJ. 2017. A smartphone-based diagnostic platform for rapid detection of Zika, chikungunya, and dengue viruses. Sci. Rep. 7: 44778.

27. Lanciotti RS, Calisher CH, Gubler DJ, Chang GJ, Vorndam AV. 1992. Rapid detection and typing of dengue viruses from clinical samples by using reverse transcriptase-polymerase chain reaction. J. Clin. Microbiol. 30: 545-551. 